\title{
The role of telehealth in supporting mothers and children during the COVID-19 pandemic
}

\author{
AUTHORS \\ WENDY SMITH RN ${ }^{1 \wedge}$ \\ SARAH TAKI PhD1,2,3 \\ LI MING WEN MD MMed PhD1,2,3,4
}

\section{CORRESPONDING AUTHOR}

LI MING WEN Population Health Research \& Evaluation Hub, Sydney Local Health District, Level 9 North, King George V Building, Camperdown NSW 2050. Phone: +61295159078

Email: Liming.Wen@health.nsw.gov.au
1 Health Promotion Unit, Population Health Research \& Evaluation Hub, Sydney Local Health District, New South Wales, Australia

2 Sydney School of Public Health, Faculty of Medicine and Health, The University of Sydney, New South Wales, Australia

3 NHMRC Centre of Research Excellence in the Early Prevention of Obesity in Childhood (EPOCH), New South Wales, Australia

4 Sydney Institute for Women, Children and Their Families, Sydney Local Health District, New South Wales, Australia

$\wedge$ On behalf of Research Nurses: Christine Phillis, Annmaree Lavery, Trisha Cant and Nada Carroll
The COVID-19 (from 'severe acute respiratory syndrome coronavirus 2') outbreak has drastically changed from epidemic to pandemic. This has led the New South Wales (NSW) Government to decrease or suspend some health services in an effort to contain the spread of the virus. The government has taken various actions to protect staff, patients, and the community against any potential transmission of COVID-19.

Child and Family Health Nurse (CFHN) services provide support, education, and information on child development, feeding and nutrition, sleep and settling techniques, and maternal (parental) emotional wellbeing to families of children aged zero to five years. Traditionally, the services are delivered face-to-face in home or community health centres. However, due to the pandemic, current services have been reduced to contain and prevent the spread of COVID-19.

Over the past three years, Sydney Local Health District Health Promotion Unit and Community Health Services have been conducting a world first three-arm randomised controlled trial testing the effectiveness of Communicating Healthy Beginnings Advice by Telephone (CHAT) ${ }^{1,2}$ to replicate the effects of a successful home-based Healthy Beginnings early obesity prevention program.3.4 Since February 2017, the CHAT study has been conducted with a total of 1,155 women recruited in the third trimester of pregnancy in NSW, Australia. The CHAT study was delivered by CFHNs via telephone or text messaging for the purpose of examining the impacts of both telephone and SMS support on a range of infants' health behaviours in the first years of life. The study findings have demonstrated positive effects on healthy infant feeding practices, reduced screen time, and co-benefits on maternal emotional wellbeing. ${ }^{5}$ In addition, the findings identified that mothers reported high satisfaction with the telephone calls and the information support delivered as part of the broader program.

This CHAT tele-support service has illustrated the potential to engage hard to reach populations for example, socially isolated women living in regional areas, women of low socioeconomic status, women with English as a second language and women with mental health issues. The service also reached women across NSW from urban to regional, and those who temporarily moved interstate and abroad. It offered effective nursing care and support to mothers and babies within their own home. The CHAT program was delivered at opportunistic moments to address key milestones of the child's growth from third trimester until two years of age. It offered convenience to the mother as it was delivered at a time when it suited her and her child. It was not reliant on availability of transportation, location or weather conditions. Flexibility was offered to continue supporting women who returned to work, and offered after hours or weekend telephone calls. 
As the COVID-19 outbreak spreads, the need for mothers and their children to home isolate may become greater on a global scale. The findings of this study have the potential to be implemented across other settings in various countries. The high rate of telephone ownership globally regardless of socio demographic characteristics may provide access to evidence-based telehealth nursing care.

The role of telehealth in supporting the community has become increasingly important during the COVID-19 pandemic as we found coincidently when delivering the Healthy Beginnings program over the phone. We found that study participants were more vulnerable to having potential anxiety and depression due to social isolation and misinformation about the virus. The telephone support calls required significantly more time to deal with complicated issues. The need for telehealth support has become paramount during the current pandemic situation. It is evident that such telephone support services could be an alternate model to face-to-face service delivery during infectious disease outbreaks that impact the feasibility of traditional approaches. There is a compelling need to integrate telehealth into existing health services.

The CHAT project was part of the Translational Research Grants Scheme, and the evidence from the study can be used to advocate to leaders working within relevant health services and policy makers on the importance of delivering telehealth. It should be emphasised that this process may involve reorienting health services to meet varying community needs. The benefits of telehealth nursing care include flexibility, nurse-initiated appointment, rapport building with participants, reaching out to socially isolated and culturally and linguistically diverse populations, and allowing more time for discussion around mother and child health issues. On the other hand, the face-to-face nursing care can be more focused on clinical health surveillance and physical health checks.

Telehealth could well be the remedy to the current crisis we face in delivering effective care while not being able to offer face-to-face health services. We would urge sustainable action to be taken to integrate telehealth into existing health services in Australia and abroad.

Acknowledgements: The CHAT study is a partnership project funded by NSW Ministry of Health Translational Research Grants Scheme 2016. We sincerely thank participating partners of the project from the Sydney, South Eastern Sydney, South Western Sydney and Southern NSW Local Health Districts, and members of the advisory committee, executive committee and project team.

Competing interests: No relevant disclosures.

\section{REFERENCES}

1. Wen LM, Rissel C, Baur LA, Hayes A, Xu H, Whelan A, Hua M, Shaw M, Phongsavan P. A 3-arm randomised controlled trial of communicating Healthy Beginnings advice by telephone (CHAT) to mothers with infants to prevent childhood obesity. BMC Public Health. 2017; 17:79

2. Wen $L M$, Rissel $C, X u H$, et al. Linking two randomised controlled trials for Healthy Beginnings(): optimising early obesity prevention programs for children under 3 years. BMC Public Health. 2019;19: 739.

3. Wen LM, Baur LA, Simpson JM, Rissel C, Flood VM. Effectiveness of an early intervention on infant feeding practices and "tummy time": randomized controlled trial. Arch Pediatr Adolesc Med. 2011;165: 701-7.

4. Wen LM, Baur LA, Simpson JM, Rissel C, Wardle K, Flood VM Effectiveness of a home-based early intervention on children's $\mathrm{BMI}$ at age two years: randomised controlled trial. BMJ. 2012; 344:e3732.

5. Wen LM, Rissel C, Xu H, Taki S, Buchanan L, Bedford K, Phongsavan P, Baur LA. Effects of telephone support and short message service on infant feeding practices, 'tummy time' and screen time at 6 and 12 months of child age: a 3-arm randomized controlled. JAMA Pediatr. Published online April 13, 2020. https://doi.org/10.1001/jamapediatrics.2020.0215 